\title{
Remotely tuneable optical filter based on polymer fibre Bragg grating
}

\author{
W. Zhang ${ }^{*}$, D. J. Webb \\ Photonics Research Group, Aston University, Birmingham, B4 7ET, UK, \\ G.-D. Peng \\ School of Electrical Engineering and Telecommunications, University of New South Wales, Sydney, \\ NSW 2052, Australia
}

\begin{abstract}
We propose a remotely tuneable optical Bragg grating filter written in polymer optical fibre (POF). Fibre optical pumping in the fibre's absorption bands increases the fibre temperature, which causes a negative wavelength change of the POF Bragg grating. By choosing a proper pumping wavelength remote tuning of the optical filter can be readily realized without changing the gain of the optical signal.
\end{abstract}

Keywords: Polymer optical fibre, fibre Bragg grating, optical pumping, remote tuning, PMMA, molecular vibration, thermal equilibrium

\section{INTRODUCTION}

BRAGG gratings in silica optical fibre have found a wide range of applications in dispersion compensation, add-drop wavelength-division multiplexing (WDM), optical signal processing and optical sensing, etc. Tuneable fibre grating devices are of interest in adaptive fibre sensor applications and spectral sensing based gas and chemical sensor applications. Remote operation would be a preferred feature for utilisation within sensor networks.

Generally speaking, techniques to control the spectral characteristics of the fibre grating structure include using a thermal head, a piezoelectric stack as well as local compression. These external tuning mechanisms generally tend to be anisotropic, leading to undesirable birefringence effects ${ }^{[1]}$. The mechanical fixtures and components required can further compromise the size and practicality. More importantly, the implementations of such schemes for remote operation are unrealistic. A tuneable filter with its actual tuning mechanism lying within the fibre itself would be a great advantage. There have been some proposed tuneable filters in which a fibre grating is inscribed on active fibre and the Bragg

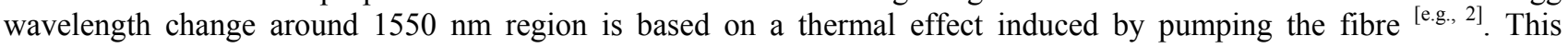
consequently gives rise to a change of the grating reflectivity.

Bragg gratings have also been inscribed into polymer optical fibre ${ }^{[3,4]}$. Within the telecommunications industry, POF has a reputation for being low cost and having high propagation loss. However, the physical and chemical properties of polymeric materials are rather different to silica, which makes it also interesting for researchers to exploit in devices for sensing applications.

\section{PRINCIPLE OF OPERATION}

The inscription of Bragg gratings has mainly been demonstrated in PMMA-based POF, where losses are much higher than with silica fibre. There are two different sources of loss in POF. Intrinsic losses are due to either absorption by the constituent material or Rayleigh scattering; both of which are dependent upon the composition of the fibre and cannot be eliminated. The second loss type is extrinsic loss, associated with structural imperfections. The absorption losses are primarily related to the molecular absorption of $\mathrm{C}-\mathrm{H}, \mathrm{N}-\mathrm{H}$, and $\mathrm{O}-\mathrm{H}$ groups in addition to absorption from electronic transitions between different energy levels within the long chain molecular bonds. Very strong absorption bands related to higher harmonics of vibrations of the $\mathrm{C}-\mathrm{H}$ bond dominate throughout the visible and near infrared ${ }^{[5]}$. It is well known

*w.zhang@aston.ac.uk

21st International Conference on Optical Fiber Sensors, edited by Wojtek J. Bock, Jacques Albert, Xiaoyi Bao, Proc. of SPIE Vol. 7753, 77537B · C 2011 SPIE · CCC code: 0277-786X/11/\$18 · doi: 10.1117/12.885917 
that molecular vibration in substances generate heat, which is referred to as the thermal effect of molecular vibration. This means that a large part of the absorption of optical energy in those spectral bands will convert into thermal energy and can be used to modulate the refractive index of the fibre. Based on this feature the characteristic wavelength of a Bragg grating written in this type of fibre can be tuned by using an additional optical pump source within those bands.

\section{EXPERIMENT AND DISCUSSION}

The operation and measurement set-up of the proposed filter is as shown in Fig. 1. The POF grating was fabricated by illuminating from above a phase mask of period $1.057 \mu \mathrm{m}$ placed on top of PMMA based single-mode POF using 325 $\mathrm{nm}$ light from a HeCd laser, which produced a Bragg wavelength of about $1569 \mathrm{~nm}$. The grating was then attached to a single mode silica fibre down-lead using UV curable glue. As mentioned before, for PMMA there are many absorption bands in the region of the visible and near infrared. To take advantage of commercial optical fibre products and achieve low loss remote operation, a pump laser at $1480 \mathrm{~nm}$ was used to induce the thermal effect in the POF grating. The reflection of the POF grating was monitored by using an IBSEN I-MON 400 wavelength interrogation system. Since the wavelength of the pump laser was located far away from the grating wavelength, the pump laser power will not affect the strength of grating reflection.

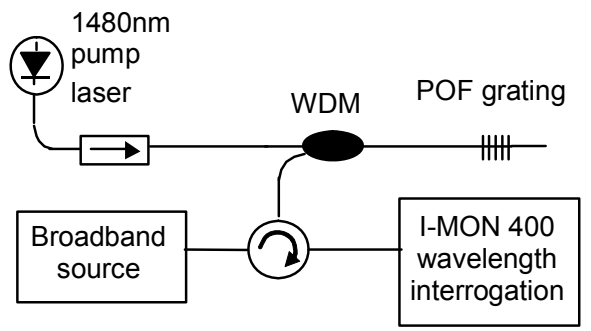

Fig. 1: Setup for measuring the device

The POF grating reflection spectrum was captured before turning on the pump laser, as shown in Fig. 2. With increasing pump laser power, the characteristic wavelength of the grating shifts towards the shorter wavelength side. Note that the temperature sensitivity of the POF grating shows the opposite sign to silica grating. This is because PMMA has a negative thermo-optic coefficient ${ }^{[6]}$ which dominates the thermal expansion of the fibre. For the POF grating used here the temperature sensitivity is around $-55 \mathrm{pm} /{ }^{\circ} \mathrm{C}$. For pump current below $30 \mathrm{~mW}$, the induced wavelength change is insignificant. As the pump current increases beyond $30 \mathrm{~mA}$ the pump laser starts lasing and the peak wavelength shifts proportionately with increasing pump power. In Fig. 2 is also shown the captured spectrum of the grating shortly after the pump current was set to $150 \mathrm{~mA}$. The wavelength change induced by the pumping is $0.315 \mathrm{~nm}$.

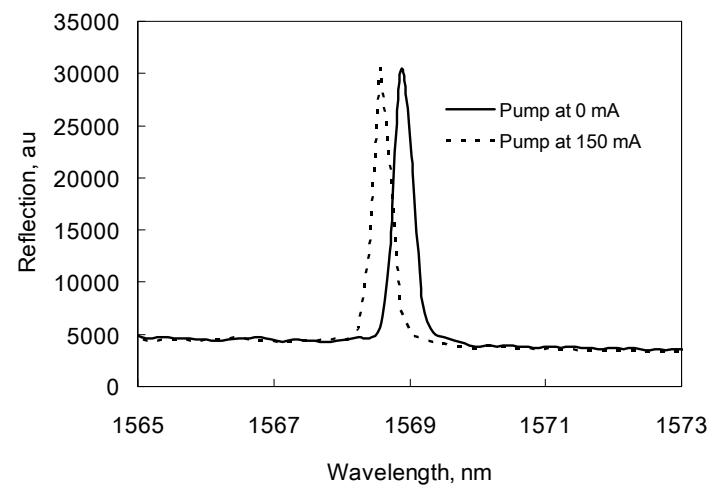

Fig. 2: Captured grating spectra

By varying pump current one can establish the relation between pump power and the induced wavelength change of the POF grating. The pump laser was set to different currents while the I-MON 400 was tracking the wavelength change of the grating. An example of the wavelength change of the grating with different pump currents is shown in Fig. 3, in 
which the pump laser was turned on at $50 \mathrm{~mA}, 100 \mathrm{~mA}$ and $150 \mathrm{~mA}$. It can be noticed that the wavelength change of the grating initially responds to the change of the pump power quickly. However, it takes a certain time for the system to reach the thermal equilibrium. As may be seen in Fig. 3 for the case of a pump current of $150 \mathrm{~mA}$, the grating wavelength has not stabilised 10 minutes after the pump laser is turned on. Therefore the wavelength change of $0.315 \mathrm{~nm}$ shown in Fig. 2 is an underestimated value for the pump current of $150 \mathrm{~mA}$.

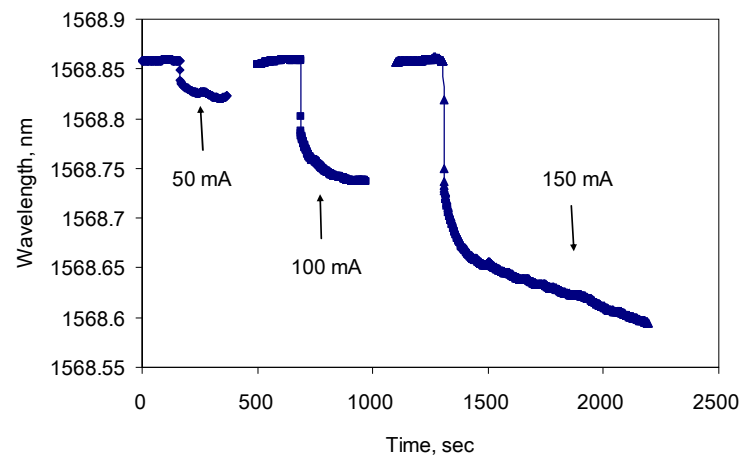

Fig. 3: Wavelength change for varying pump current

A full cycle of the wavelength change as pump current varied from $0 \mathrm{~mA}$ to $180 \mathrm{~mA}$ to $0 \mathrm{~mA}$, was observed in the experiment, the captured wavelength change being shown in Fig. 4. As the laser turned on the wavelength of the POF grating initially drops quickly but it takes about 50 minutes to reach thermal equilibrium at this pump current. The wavelength change induced can be recalculated from the figure. It shows a change of $0.5 \mathrm{~nm}$ due to the thermal effect induced by the pump laser at $180 \mathrm{~mA}$.

One can also notice from Fig. 4 that the times taken for the POF grating to reach thermal equilibrium for pumping current up and down are different. When the pump laser is turned off (from $180 \mathrm{~mA}$ to $0 \mathrm{~mA}$ ), the POF grating takes longer time to reach the equilibrium. This is because in PMMA based optical fibre the wavelength change is influenced by water content existing in the fibre. When the temperature increases, the water desorbs from the PMMA, while when the temperature decreases, the fibre adsorbs water. In PMMA the desorption process is faster than the adsorption process [7]. When pump current increases and consequently the temperature in the fibre increases, water is driven out of fibre, leading to a reduction of refractive index in fibre; when pump current decreases, a temperature reduction happens, water is adsorbed leading to a larger refractive index in fibre. A reduced fibre diameter could reduce the equilibrium time for both desorption and adsorption processes. From Fig. 4 one can also notice that the POF grating wavelength goes back to its initial value after the pump laser is turned off. It proves there is no hysteresis for this proposed tuning technique.

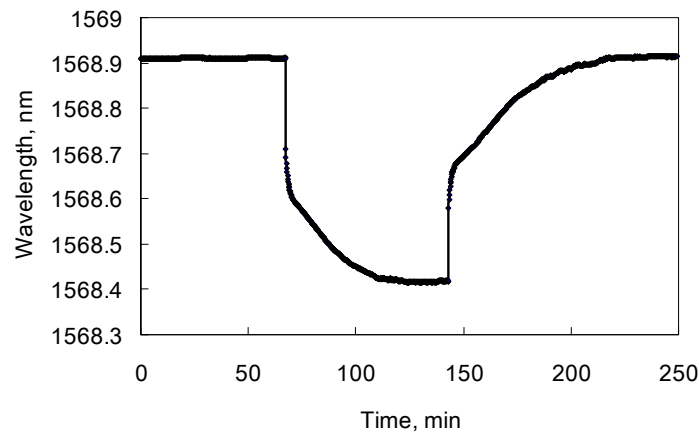

Fig. 4: Full cycle of wavelength change for pump current of $180 \mathrm{~mA}$ 
The wavelength change of the grating at different pump currents was measured at the completion of thermal equilibrium and is shown in Fig. 5. The pump power at the different pump currents was measured at the output port of the WDM coupler, also shown in Fig. 5. This only provides an estimation of the pump power applied onto the POF grating due to the insertion loss induced by the mismatch of fibre mode sizes and UV gluing. It can be seen from Fig. 5 that after lasing threshold both pump power and Bragg wavelength change show fairly good linearity with the pump current. By ignoring the connection induced insertion loss we then have an estimated tuning sensitivity of $-56 \mathrm{pm} / \mathrm{mW}$.

Since the temperature sensitivity of the POF grating is much higher than that of a silica fibre grating one can notice some drift of the characteristic wavelength of the POF grating used in the experiments due to the change of the surrounding temperature. The reflected optical intensity from the grating is little affected by the varying pump power since the pump laser wavelength is fairly distant from the grating characteristic wavelength.

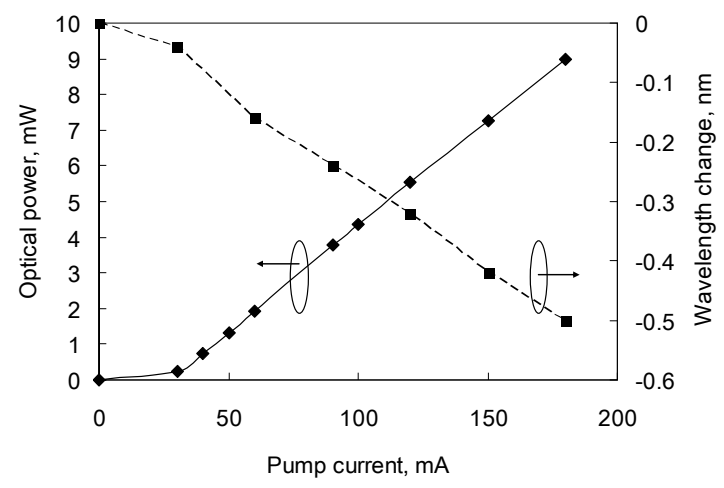

Fig. 5: Pump power and wavelength change at different pump current

\section{CONCLUSIONS}

An optical pump controlled, remotely tuneable fibre filter using a polymer optical fibre grating has been investigated. The proposed technique exhibits a large tuning sensitivity and range and is highly repeatable without hysteresis. It offers a simple and effective means to implement a remotely tuneable fibre grating filter for different applications.

\section{REFERENCES}

[1] de Matos, C.J.S. Torres, P. Valente, L.C.G. Margulis, W. Stubbe, R., "Fibre Bragg grating (FBG) characterization and shaping by local pressure," J. Lightwave Technol., 19(8), 1206-1211, (2001).

[2] Lai , Y., Zhang, W., Zhang, L., Williams, J. A. R., and Bennion, I., "Optically tunable fibre grating transmission filters," Opt. Lett., 28(15), 2446-2448 (2003).

[3] Xiong, Z., Peng, G.D., Wu, B., Chu, P.L., "Highly tunable Bragg gratings in single-mode polymer optical fibres," IEEE Photon. Tech. Lett., 11(3), 352-354 (1999)

[4] Dobb, H., Webb, D. J., Kalli, K., Argyros, A., Large,M. C. J., and van Eijkelenborg, M. A., "Continuous wave ultraviolet light-induced fibre Bragg gratings in few- and single-mode microstructured polymer optical fibres," Opt. Lett., 30(24), 3296-3298 (2005)

[5] Pitois, C., Hult, A., Wiesmann, D., "Absorption and scattering in low-loss polymer optical waveguides," J. Opt. Soc. Am. B, 18(7), 908-912 (2001)

[6] Liu, H.Y. Peng, G.D. Chu, P.L., "Thermal tuning of polymer optical fibre Bragg gratings," IEEE Photon. Tech. Lett., 13(8), 824-826 (2001).

[7] Turner, D. T., "Polymethyl methacrylate plus water: sorption kinetics and volumetric changes," Polymer, 23, $197-$ $202,(1982)$ 